\title{
A longitudinal study assessing differences in causes of death among housed and homeless people diagnosed with HIV in San Francisco
}

Nancy A. Hessol ${ }^{1 *}$ (D, Monica Eng ${ }^{1}$, Annie Vu², Sharon Pipkin², Ling C. Hsu² and Susan Scheer ${ }^{2}$

\begin{abstract}
Background: San Francisco has implemented several programs addressing the needs of two large vulnerable populations: people living with HIV and those who are homeless. Assessment of these programs on health outcomes is paramount for reducing preventable deaths.

Methods: Individuals diagnosed with HIV/AIDS and reported to the San Francisco Department of Public Health HIV surveillance registry, ages 13 years or older, who resided in San Francisco at the time of diagnosis, and who died between January 1, 2002, and December 31, 2016 were included in this longitudinal study. The primary independent variable was housing status, dichotomized as ever homeless since diagnosed with HIV, and the dependent variables were disease-specific causes of death, as noted on the death certificate. The Cochran-Armitage test measured changes in the mortality rates over time and unadjusted and adjusted Poisson regression models measured prevalence ratios (PR) and 95\% confidence intervals (Cl) for causes of death.

Results: A total of 4158 deceased individuals were included in the analyses: the majority were male (87\%), ages 40-59 years old at the time of death (64\%), non-Hispanic White (60\%), men who have sex with men (54\%), had an AIDS diagnosis prior to death (87\%), and San Francisco residents at the time of death (63\%). Compared to those who were housed, those who were homeless were more likely to be younger at time of death, African American, have a history of injecting drugs, female or transgender, and were living below the poverty level (all $p$ values $<0.0001$ ). Among decedents who were SF residents at the time of death, there were declines in the proportion of deaths due to AIDSdefining conditions $(p<0.05)$ and increases in accidents, cardiomyopathy, heart disease, ischemic disease, non-AIDS cancers, and drug overdoses $(p<0.05)$. After adjustment, deaths due to mental disorders (aPR $=1.63,95 \% \mathrm{Cl} 1.24,2.14)$ were more likely and deaths due to non-AIDS cancers ( $\mathrm{aPR}=0.63,95 \% \mathrm{Cl} 0.44,0.89)$ were less likely among those experiencing homelessness.
\end{abstract}

Conclusions: Additional efforts are needed to improve mental health services to homeless people with HIV and prevent mental-health related mortality.

Keywords: Cause of death, HIV, Homeless, Housing, Mental health, Mortality

\footnotetext{
* Correspondence: Nancy.Hessol@ucsf.edu

${ }^{1}$ Department of Clinical Pharmacy, University of California, 3333 California

Street, Suite 420, San Francisco, CA 94118, USA

Full list of author information is available at the end of the article
}

(c) The Author(s). 2019 Open Access This article is distributed under the terms of the Creative Commons Attribution 4.0 International License (http://creativecommons.org/licenses/by/4.0/) which permits unrestricted use, distribution, and reproduction in any medium, provided you give appropriate credit to the original author(s) and the source, provide a link to the Creative Commons license, and indicate if changes were made. The Creative Commons Public Domain Dedication waiver (http://creativecommons.org/publicdomain/zero/1.0/) applies to the data made available in this article, unless otherwise stated. 


\section{Background}

Use of effective antiretroviral therapy (ART) has enabled many people living with HIV (PLWH) to have healthier and longer lives. In San Francisco the three-year survival time among people with stage 3 HIV (AIDS) increased from $52 \%$ in the pre-ART years of $1990-1995$ to $90 \%$ in the ART era years of 2006-2015 [1]. Adoption of ART has also led to a change in the causes of death among PLWH. This change is evident in San Francisco where the proportion of deaths among PLWH in which HIV was listed as a cause of death declined from $69.8 \%$ in the years 2006-2009 to 59.0\% in 2014-2017 [1]. Conversely, deaths due to heart disease and non-AIDS cancer increased from 23.2 and $14.7 \%$, respectively, to 29.9 and $20.3 \%$, respectively, for these same time periods.

While cause-specific mortality among PLWH is well studied, there is little information on cause-specific mortality for homeless PLWH. In 2016 there were an estimated 291 homeless PLWH in San Francisco [1]. A city-wide point-in-time count in 2017 estimated that 4353 people, regardless of HIV status, were unsheltered in San Francisco and another 2505 individuals were marginally sheltered [2]. Separate studies of people living in Philadelphia, New York City, and Boston have reported that homeless individuals have higher mortality rates compared to the general population due to factors such as higher rates of psychiatric illness and substance abuse [3-5]. Carrying the additional burden of living with HIV puts people living with homelessness in a more vulnerable state.

Despite these comorbidities, the five-year HIV survival rate has improved over time for PLWH who are experiencing homelessness in San Francisco. Among all PLWH in San Francisco diagnosed with HIV from 2002 to 2006, the five-year survival rate for homeless individuals was $79 \%$ compared to $92 \%$ among housed individuals $(p<0.001)$ [6]. However, among persons diagnosed with HIV more recently from 2007 to 2011 there was no difference in the five-year survival rate between homeless and housed people $(p=0.40)$ [6]. A study analyzing a permanent supportive housing program in San Francisco found that among 6558 AIDS cases diagnosed from 1996 to 2006 in San Francisco, the five-year survival rate for persons who were homeless was $67 \%$ compared to $81 \%$ among those who were housed under permanent supportive housing $(p<0.0001)$ [7]. In contrast to the improvement in mortality data, a 2017 study in San Francisco reported poorer HIV viral suppression among homeless PLWH. The study observed lower odds for achieving viral suppression with individuals living in shelters (adjusted odds ratio $[\mathrm{OR}]=0.26,95 \%$ CI $0.12,0.59$ ) and those living outdoors (adjusted OR $=0.15,95 \% \mathrm{CI}$ $0.08,0.29$ ) [8]. Even though several efforts have been made to provide stable housing and resources in San
Francisco, homelessness continues to be a barrier to managing an HIV diagnosis.

Three interventions aimed to support persons experiencing homelessness in San Francisco were established between 1998 and 2006: Direct Access to Housing (DAH), Care Not Cash, and Mental Health Services Act (MHSA). DAH, also known as permanent supportive housing, was established in 1998 by the San Francisco Department of Public Health (DPH) Housing and Urban Health Section. The program aims to house low-income San Francisco residents who are homeless and have special needs such as mental health issues, alcohol or substance abuse, or complex medical conditions such as AIDS [9]. Additionally, the Care Not Cash program was established by the San Francisco Human Services Agency in 2004 and shifted the utilization of funds from cash grants given to the homeless to expanding mental health and substance abuse services [10]. The budget for such services and treatment beds saw an increase from $\$ 666,000$ in 2004 to $\$ 1.2$ million in 2008 and also led to an additional 1321 affordable units for the homeless [10]. Furthermore, in 2006, the California Department of Mental Health implemented MHSA, which added a state personal income tax surcharge of $1 \%$ on taxpayers with annual taxable incomes of more than $\$ 1$ million (approximately 25,000 to 30,000 taxpayers) [11]. These funds were transferred to the new Mental Health Services Fund and were used to expand the county medical services such as psychiatric, counseling, and hospitalization to any Californians who lacked coverage for such care, as well as permanent supportive housing for homeless individuals with serious mental disorders [12].

Along the same time period that these programs were established, HIV prevention and care leaders in San Francisco also implemented programs to improve HIV care. In May 2006, San Francisco General Hospital (SFGH; San Francisco's only municipal hospital) eliminated the requirement for written consent for HIV testing and added the HIV antibody test to the routine lab order form [13]. In 2010, the San Francisco DPH implemented universal Test and Treat in all publicly funded clinics, recommending that all patients initially diagnosed with HIV be offered ART regardless of their CD4 T lymphocyte (CD4) count. Prior to this policy, ART was not universally prescribed to patients with CD4 counts above 500 cells $/ \mathrm{mm}^{3}$. A study evaluating the intervention of Test and Treat policies demonstrated significant increases of viral suppression at the population [14]. The SFGH's Rapid ART Program Initiative for New Diagnosis (RAPID) began in 2013 and established that any newly diagnosed or newly re-engaged patient would see an HIV health team, be offered ART (regardless of CD4 count), receive counseling, and agree on a sustainable care plan on the same day of their diagnosis/ 
re-engagement, or within two to five days [15]. The success of the RAPID program at SFGH led to the Citywide RAPID program in 2015.

Given that San Francisco has provided many housing programs and supportive services to reduce the number of people who are unsheltered and that San Francisco was an early adopter of better clinical care programs for people living with HIV, we aimed to identify differences in causes of death (by calendar period) between people with HIV who were housed and those who were homeless to help understand the impact of these programs on fatal health outcomes. With the improvement in HIV care programs, we hypothesized that HIV-related causes of death, AIDS-defining opportunistic infections, and AIDS-defining cancers would be similar among PLWH who experienced homelessness and those who were housed. In addition, we hypothesized that deaths due to substance abuse and mental health disorders would be higher among homeless compared to housed PLWH.

\section{Methods}

\section{Study sample and data collection}

Included in the analyses were San Francisco residents over 12 years old, diagnosed with HIV or AIDS and reported to the San Francisco DPH, and who died from January 1, 2002 through December 31, 2016. Sociodemographic and HIV transmission characteristics, AIDSrelated clinical data, and vital status were collected as part of routine HIV surveillance on all study participants. Computer matches with the National Death Index Plus provided information on multiple coded causes of death. HIV surveillance data collection is required by state law (California Health and Safety Code (HSC) 121,022 and HSC 120130), therefore Institutional Review Board (IRB) approval and consent was deemed unnecessary according to state regulations. Data used in this study is protected by state law and is not publicly available.

The International Classification of Diseases 10th edition [16] was used to code the cause of death information from death certificates. All coded causes and conditions contributing to death (including the underlying cause of death), as listed on the death certificate, were included in our multiple cause of death category. We included the most frequently occurring causes of death, both HIV/ AIDS-related (presented as all HIV/AIDS-related deaths and separately as AIDS-related malignancies and AIDSrelated opportunistic infections) and non-HIV-related, as our dependent variable. We also included the following causes of death regardless of the frequency of their occurrence because they are more likely to occur in people who are homeless: accident, assault, suicide, drug-related overdose, alcohol-related liver disease, and mental disorders. For AIDS-defining opportunistic infections [17], any HIV/ AIDS cause of death, AIDS-defining and non-AIDS- defining cancers, heart disease (including cardiomyopathy, cerebrovascular, and ischemic disease), and mental disorders (including mental disorders due to substance abuse) composite categories were created.

Most socio-demographic characteristics in our analyses included those obtained at the time of HIV diagnosis, except for age at death, county of death, and housing status. For the purpose of HIV surveillance, the Centers for Disease Control and Prevention (CDC) uses the federal definition of homelessness. Thus, an individual is defined as homeless if their medical record denotes that the patient is not housed or homeless at the time of HIV or AIDS diagnosis, or the person's address at diagnosis is a free postal address not connected to a residence (i.e., general delivery) or a known homeless shelter [1]. Not included in this definition are individuals with unstable (living with friends) or marginal housing (living in a single room occupancy units) [1]. For our analyses, a person was defined as homeless if periodic review of the medical record noted that the patient was homeless at the time of HIV diagnosis, AIDS diagnosis, at follow up, or at death [1].

In contingency table analyses, age at death was categorically defined by decades: $20-29,30-39,40-49$, $50-59,60-69$, and 70-79. Race was categorized as nonHispanic White, Hispanic, African-American, or other, including multi-race/ethnicity. HIV transmission risk category was categorized as men who have sex with men (MSM), persons who inject drugs (PWID), men who have sex with men and who also inject drugs (MSMPWID), heterosexual, or other. Gender was classified as male, female, or transgender. Living below the federal poverty level at time of diagnosis was defined as having lived in a census tract where more than $20 \%$ of persons aged 18 years or older had a median annual household income that was below the United States poverty level [18]. Country of birth was dichotomized as USA/US Dependency or other and county of residence at death was dichotomized as San Francisco or other. Prescription of ART was dichotomized as yes or no.

\section{Statistical analyses}

The primary independent variable was housing status dichotomized as homeless at or after HIV diagnosis or not. The primary dependent variable was multiple condition or disease-specific cause of death.

The distribution of case characteristics by housing status was compared using contingency tables, and $p$ values were calculated using chi-square and Cochran-Armitage trend tests. This method was also used to explore the differences in causes of death among PLWH stratified by county of residency at time of death (San Francisco vs. non-San Francisco residents), which serves as a surrogate measure for access to San Francisco specific interventions. 
Table 1 Study characteristics among people diagnosed with HIV in San Francisco who died in 2002-2016

\begin{tabular}{|c|c|c|c|c|}
\hline & $\begin{array}{l}\text { Homeless } \\
N=559\end{array}$ & $\begin{array}{l}\text { Housed } \\
N=3599\end{array}$ & & \\
\hline Characteristic & n (\%) & n (\%) & Chi Square $p$ value & Trend Test $p$ value \\
\hline \multicolumn{5}{|l|}{ Year of Death } \\
\hline $2002-2004$ & $148(26.48)$ & $845(23.48)$ & 0.3099 & 0.8104 \\
\hline $2005-2007$ & $115(20.57)$ & $869(24.15)$ & & \\
\hline 2008-2010 & $103(18.43)$ & 646 (17.95) & & \\
\hline $2011-2013$ & $93(16.64)$ & $623(17.31)$ & & \\
\hline 2014-2016 & $100(17.89)$ & $616(17.12)$ & & \\
\hline \multicolumn{5}{|l|}{ Age at death } \\
\hline $20-29$ & $20(3.58)$ & $39(1.08)$ & $<0.0001$ & \\
\hline $30-39$ & $96(17.17)$ & $349(9.70)$ & & \\
\hline $40-49$ & $221(39.53)$ & $1084(30.12)$ & & \\
\hline $50-59$ & $165(29.52)$ & $1211(33.65)$ & & \\
\hline $60-69$ & $50(8.94)$ & $657(18.26)$ & & \\
\hline $70-99$ & $7(1.25)$ & $259(7.20)$ & & \\
\hline \multicolumn{5}{|l|}{ Race } \\
\hline Non-Hispanic White & $250(44.72)$ & $2227(61.88)$ & $<0.0001$ & \\
\hline Hispanic & $76(13.60)$ & $441(12.25)$ & & \\
\hline African-American & $196(35.06)$ & $686(19.06)$ & & \\
\hline Other & $37(6.62)$ & $245(6.81)$ & & \\
\hline \multicolumn{5}{|l|}{ Transmission Risk Factor } \\
\hline MSM & $92(16.46)$ & $2146(59.63)$ & $<0.0001$ & \\
\hline MSM-PWID & $205(36.67)$ & $789(21.92)$ & & \\
\hline PWID & $235(42.04)$ & $508(14.12)$ & & \\
\hline Heterosexual & $19(3.40)$ & $86(2.39)$ & & \\
\hline Other & $8(1.43)$ & 70 (1.94) & & \\
\hline \multicolumn{5}{|l|}{ Gender } \\
\hline Female & $108(19.32)$ & $287(7.97)$ & $<0.0001$ & \\
\hline Male & $411(73.52)$ & $3206(89.08)$ & & \\
\hline Transgender & $40(7.16)$ & $106(2.95)$ & & \\
\hline \multicolumn{5}{|l|}{ Poverty at Diagnosis } \\
\hline Yes & $505(90.34)$ & $837(23.26)$ & $<0.0001$ & \\
\hline No & $54(9.66)$ & $2762(76.74)$ & & \\
\hline \multicolumn{5}{|l|}{ Country of Birth } \\
\hline USA/US Dependency & $502(89.80)$ & $3189(88.61)$ & 0.4050 & \\
\hline Other & $57(10.20)$ & $410(11.39)$ & & \\
\hline \multicolumn{5}{|c|}{ County of Residence at Death } \\
\hline San Francisco & $378(67.62)$ & $2253(62.60)$ & 0.0220 & \\
\hline Other & $181(32.38)$ & $1346(37.40)$ & & \\
\hline AIDS dx prior to death & $483(86.40)$ & $3139(87.22)$ & 0.5929 & \\
\hline \multicolumn{5}{|l|}{ Prescribed ART } \\
\hline Yes & $424(75.85)$ & $3024(84.02)$ & $<0.0001$ & \\
\hline No & $135(24.15)$ & $575(15.98)$ & & \\
\hline
\end{tabular}

First CD4 Count after Diagnosis (Within 6 months) 
Table 1 Study characteristics among people diagnosed with HIV in San Francisco who died in 2002-2016 (Continued)

\begin{tabular}{|c|c|c|c|c|}
\hline & $\begin{array}{l}\text { Homeless } \\
N=559\end{array}$ & $\begin{array}{l}\text { Housed } \\
N=3599\end{array}$ & & \\
\hline Characteristic & n (\%) & n (\%) & Chi Square $p$ value & Trend Test $p$ value \\
\hline CD4 $<200$ & $128(34.32)$ & $845(36.84)$ & 0.1450 & \\
\hline CD4 200-499 & $139(37.27)$ & $905(39.45)$ & & \\
\hline CD4 $>500$ & $106(28.42)$ & $544(23.71)$ & & \\
\hline Missing data & 186 & 1305 & & \\
\hline \multicolumn{5}{|c|}{ First Viral Load after Diagnosis (within 6 months) } \\
\hline Viral load $<401$ & $22(8.33)$ & $113(8.96)$ & 0.7610 & \\
\hline Viral load 401-3999 & $46(17.42)$ & $207(16.42)$ & & \\
\hline Viral load 4000-49,999 & $102(38.64)$ & $454(36.00)$ & & \\
\hline Viral load $>49,000$ & $94(35.61)$ & $487(38.62)$ & & \\
\hline Missing data & 295 & 2338 & & \\
\hline
\end{tabular}

$P$ values $<0.05$ are shown in bold

To compare differences in cause-specific deaths between the housed and the homeless populations, unadjusted and adjusted Poisson regression models for binary outcomes were performed to calculate the prevalence ratios (PR's), $p$ values, and 95\% confidence intervals (CI's). The binary explanatory variable for the unadjusted model was housing status, with housed individuals being the reference group. We considered a $\mathrm{p}$ value of less than 0.05 significant. Adjusted models were constructed for each cause of death if the housing variable resulted in a statistically significant PR in the unadjusted model.

Adjusted regression models controlled for the following factors with their respective reference group noted in parentheses: gender (male), race (non-Hispanic white), a concurrent initial diagnosis of HIV and AIDS (yes or no), HIV transmission category (MSM), low income (yes or no), and county of residence at death (San Francisco). In addition, the regression models were adjusted for age, which was continuous per decade, and year of death, which was continuous per year. All statistical analyses were performed using SAS ${ }^{\circ}$ software version 9.4 [19].

\section{Results}

\section{Study sample characteristics}

A total of 4158 deceased individuals were included in the analyses: the majority were male (87\%), ages $40-59$ years old at the time of death (64\%), non-Hispanic White (60\%), MSM (54\%), had an AIDS diagnosis prior to death $(87 \%)$, and San Francisco residents at the time of death (63\%; Table 1). Compared to those who were housed, those who were homeless were more likely to be younger at time of death, African American, PWID, female or transgender, and living below the poverty level, and less likely to have been prescribed ART (all $p$ values $<0.0001$; Table 1). Those with missing baseline CD4 cell count and HIV viral load were more likely to be housed than homeless ( $p=0.17$ and $p<0.0001$, respectively).

Causes of deaths by county of residency at time of death Both San Francisco residents and non-San Francisco residents had a significant decline in deaths due to HIV/AIDS and pneumonia and a significant increase in deaths due to heart disease, ischemic disease, and non-AIDS cancer. San Francisco residents had a decrease in trend for AIDS cancer, AIDS opportunistic infections, non-cancer AIDS opportunistic infections and an increase in trend for accidental deaths, cardiomyopathy, and overdose. Non-San Francisco residents had an increase in deaths due to assault, COPD, diabetes, and mental disorders (all $p$ values $<0.0001$; Table 2).

\section{Prevalence ratios - multiple causes of death}

The unadjusted PR showed that homeless individuals were more likely to die from an accident, assault, mental disorder, mental disorder due to substance abuse use, overdose, and viral hepatitis compared to housed individuals. On the other hand, homeless individuals were less likely to die from diabetes, ischemic heart disease, and non-AIDS cancers.

Adjusted Poisson regression identified two cause of death categories that resulted in significantly higher adjusted prevalence ratios (aPRs) for homelessness when compared to housed individuals: mental disorders $(\mathrm{aPR}=1.63,95 \%$ CI 1.24, 2.14; Table 3$)$ and the sub-category mental disorders due to substance use $(\mathrm{aPR}=1.70,95 \%$ CI 1.27, 2.27; Table 3). In addition, those with a history of homelessness were $37 \%$ less likely to die from non-AIDS cancers $(\mathrm{aPR}=0.63,95 \%$ CI 0.44, 0.89; Table 3). 
Table 2 Temporal trends in cause-specific deaths by county of residency at time of death (San Francisco County vs. all other counties), among people diagnosed with HIV in San Francisco who died in 2002-2016

\begin{tabular}{|c|c|c|c|c|c|}
\hline \multicolumn{3}{|c|}{ Cause of Death among San Francisco Residents } & \multicolumn{3}{|c|}{ Cause of Death among Non-San Francisco Residents } \\
\hline Cause of Death & $\mathrm{N}(\%)$ & $\begin{array}{l}\text { Trend Test } \\
p \text { value }\end{array}$ & Cause of Death & N (\%) & $\begin{array}{l}\text { Trend Test } \\
p \text { value }\end{array}$ \\
\hline Accidental death (including drug related) & & $<.0001$ & Accidental death (including drug related) & & 0.6497 \\
\hline $2002-2004$ & $24(3.64)$ & & $2002-2004$ & $27(8.11)$ & \\
\hline $2005-2007$ & $61(9.58)$ & & 2005-2007 & $37(10.66)$ & \\
\hline $2008-2010$ & $65(13.77)$ & & 2008-2010 & $29(10.47)$ & \\
\hline $2011-2013$ & $58(12.89)$ & & $2011-2013$ & $27(10.15)$ & \\
\hline $2014-2016$ & $60(14.56)$ & & 2014-2016 & $29(9.54)$ & \\
\hline AIDS cancer & & $<0.0001$ & AIDS cancer & & 0.1002 \\
\hline $2002-2004$ & $72(10.91)$ & & $2002-2004$ & $33(9.91)$ & \\
\hline $2005-2007$ & $60(9.42)$ & & 2005-2007 & $19(5.48)$ & \\
\hline $2008-2010$ & $33(6.99)$ & & 2008-2010 & $29(10.47)$ & \\
\hline $2011-2013$ & $28(6.22)$ & & $2011-2013$ & $20(7.52)$ & \\
\hline 2014-2016 & $19(4.61)$ & & 2014-2016 & $15(4.93)$ & \\
\hline $\begin{array}{l}\text { AIDS opportunistic infections (excluding } \\
\text { AIDS cancers) }\end{array}$ & & $<0.0001$ & $\begin{array}{l}\text { AIDS opportunistic infections (excluding } \\
\text { AIDS cancers) }\end{array}$ & & 0.0639 \\
\hline $2002-2004$ & $188(28.48)$ & & $2002-2004$ & $81(24.32)$ & \\
\hline $2005-2007$ & $153(24.02)$ & & 2005-2007 & $79(22.77)$ & \\
\hline $2008-2010$ & $98(20.76)$ & & 2008-2010 & $56(20.22)$ & \\
\hline $2011-2013$ & $79(17.56)$ & & $2011-2013$ & $47(17.67)$ & \\
\hline 2014-2016 & $73(17.72)$ & & 2014-2016 & $61(20.07)$ & \\
\hline Alcohol related liver disease & & 0.1152 & Alcohol related liver disease & & 0.4591 \\
\hline $2002-2004$ & $7(1.06)$ & & $2002-2004$ & $5(1.50)$ & \\
\hline $2005-2007$ & $6(0.94)$ & & $2005-2007$ & $3(0.86)$ & \\
\hline 2008-2010 & $6(1.27)$ & & 2008-2010 & $2(0.36)$ & \\
\hline $2011-2013$ & $6(1.33)$ & & $2011-2013$ & $0(0.00)$ & \\
\hline 2014-2016 & $9(2.18)$ & & 2014-2016 & $4(1.32)$ & \\
\hline Assault & & 0.1028 & Assault & & 0.0490 \\
\hline $2002-2004$ & $3(0.45)$ & & $2002-2004$ & $1(0.30)$ & \\
\hline $2005-2007$ & $5(0.78)$ & & 2005-2007 & $2(0.58)$ & \\
\hline 2008-2010 & $3(0.64)$ & & 2008-2010 & $0(0.00)$ & \\
\hline $2011-2013$ & $6(1.33)$ & & $2011-2013$ & $2(0.75)$ & \\
\hline 2014-2016 & $5(1.21)$ & & 2014-2016 & $5(1.64)$ & \\
\hline Cardiomyopathy & & 0.0080 & Cardiomyopathy & & 0.4488 \\
\hline $2002-2004$ & $13(1.97)$ & & $2002-2004$ & $7(2.10)$ & \\
\hline $2005-2007$ & $7(1.10)$ & & $2005-2007$ & $9(2.59)$ & \\
\hline $2008-2010$ & $11(2.33)$ & & $2008-2010$ & $7(2.53)$ & \\
\hline $2011-2013$ & $10(2.22)$ & & $2011-2013$ & $4(1.50)$ & \\
\hline 2014-2016 & $18(4.37)$ & & 2014-2016 & $5(1.64)$ & \\
\hline Cerebral vascular event & & 0.1230 & Cerebral vascular event & & 0.1221 \\
\hline $2002-2004$ & $19(2.88)$ & & $2002-2004$ & $10(3.00)$ & \\
\hline $2005-2007$ & $16(2.51)$ & & $2005-2007$ & $11(3.17)$ & \\
\hline 2008-2010 & $22(4.66)$ & & 2008-2010 & $7(2.53)$ & \\
\hline 2011-2013 & $13(2.89)$ & & 2011-2013 & $6(2.26)$ & \\
\hline
\end{tabular}


Table 2 Temporal trends in cause-specific deaths by county of residency at time of death (San Francisco County vs. all other counties), among people diagnosed with HIV in San Francisco who died in 2002-2016 (Continued)

\begin{tabular}{|c|c|c|c|c|c|}
\hline \multicolumn{3}{|c|}{ Cause of Death among San Francisco Residents } & \multicolumn{3}{|c|}{ Cause of Death among Non-San Francisco Residents } \\
\hline Cause of Death & N (\%) & $\begin{array}{l}\text { Trend Test } \\
p \text { value }\end{array}$ & Cause of Death & $\mathrm{N}(\%)$ & $\begin{array}{l}\text { Trend Test } \\
p \text { value }\end{array}$ \\
\hline $2014-2016$ & $19(4.61)$ & & 2014-2016 & $18(5.92)$ & \\
\hline COPD & & 0.1489 & COPD & & 0.0401 \\
\hline $2002-2004$ & $37(5.61)$ & & $2002-2004$ & $17(5.11)$ & \\
\hline $2005-2007$ & $48(7.54)$ & & $2005-2007$ & $7(2.02)$ & \\
\hline 2008-2010 & $39(8.26)$ & & $2008-2010$ & $12(4.33)$ & \\
\hline $2011-2013$ & $28(6.22)$ & & $2011-2013$ & $17(6.39)$ & \\
\hline 2014-2016 & $36(8.74)$ & & 2014-2016 & $21(6.91)$ & \\
\hline Diabetes & & 0.0804 & Diabetes & & 0.0003 \\
\hline $2002-2004$ & $14(2.12)$ & & $2002-2004$ & $4(1.20)$ & \\
\hline $2005-2007$ & $29(4.55)$ & & 2005-2007 & $11(3.17)$ & \\
\hline 2008-2010 & $18(3.81)$ & & 2008-2010 & $11(3.97)$ & \\
\hline $2011-2013$ & $25(5.56)$ & & $2011-2013$ & $22(8.27)$ & \\
\hline 2014-2016 & 15 (3.64) & & 2014-2016 & $16(5.26)$ & \\
\hline HIV/AIDS & & $<0.0001$ & HIV/AIDS & & $<0.0001$ \\
\hline 2002-2004 & 557 (84.39) & & $2002-2004$ & $259(77.78)$ & \\
\hline $2005-2007$ & $472(74.10)$ & & $2005-2007$ & $243(70.03)$ & \\
\hline 2008-2010 & 314 (66.53) & & 2008-2010 & $194(70.04)$ & \\
\hline $2011-2013$ & $284(63.11)$ & & $2011-2013$ & $164(61.65)$ & \\
\hline 2014-2016 & 258 (62.62) & & 2014-2016 & $183(60.20)$ & \\
\hline Heart disease & & 0.0004 & Heart disease & & $<0.0001$ \\
\hline 2002-2004 & $132(20.00)$ & & $2002-2004$ & 73 (21.92) & \\
\hline 2005-2007 & 128 (20.09) & & 2005-2007 & $79(22.77)$ & \\
\hline 2008-2010 & $132(27.97)$ & & $2008-2010$ & 79 (28.52) & \\
\hline 2011-2013 & $99(22.00)$ & & $2011-2013$ & 89 (33.46) & \\
\hline 2014-2016 & $121(29.97)$ & & 2014-2016 & $101(33.22)$ & \\
\hline Ischemic & & 0.0408 & Ischemic & & 0.0494 \\
\hline 2002-2004 & $37(5.61)$ & & 2002-2004 & $25(7.51)$ & \\
\hline $2005-2007$ & $36(5.65)$ & & $2005-2007$ & $23(6.63)$ & \\
\hline 2008-2010 & $36(7.63)$ & & $2008-2010$ & $28(10.11)$ & \\
\hline $2011-2013$ & $35(7.78)$ & & $2011-2013$ & $24(9.02)$ & \\
\hline 2014-2016 & $33(8.01)$ & & 2014-2016 & $34(11.18)$ & \\
\hline Liver disease & & 0.0539 & Liver disease & & 0.2200 \\
\hline $2002-2004$ & $111(16.82)$ & & $2002-2004$ & $52(15.62)$ & \\
\hline $2005-2007$ & 85 (13.34) & & $2005-2007$ & $41(11.82)$ & \\
\hline 2008-2010 & $67(14.19)$ & & 2008-2010 & 37 (13.36) & \\
\hline 2011-2013 & $56(12.44)$ & & 2011-2013 & $30(11.28)$ & \\
\hline 2014-2016 & $53(12.84)$ & & 2014-2016 & $37(12.17)$ & \\
\hline Mental disorders & & 0.2685 & Mental disorders & & 0.0035 \\
\hline 2002-2004 & $61(9.34)$ & & $2002-2004$ & $25(7.51)$ & \\
\hline $2005-2007$ & 74 (11.62) & & $2005-2007$ & $36(10.37)$ & \\
\hline 2008-2010 & $50(10.59)$ & & 2008-2010 & $28(10.11)$ & \\
\hline $2011-2013$ & $43(9.56)$ & & $2011-2013$ & $36(13.53)$ & \\
\hline
\end{tabular}


Table 2 Temporal trends in cause-specific deaths by county of residency at time of death (San Francisco County vs. all other counties), among people diagnosed with HIV in San Francisco who died in 2002-2016 (Continued)

\begin{tabular}{|c|c|c|c|c|c|}
\hline \multicolumn{3}{|c|}{ Cause of Death among San Francisco Residents } & \multicolumn{3}{|c|}{ Cause of Death among Non-San Francisco Residents } \\
\hline Cause of Death & $\mathrm{N}(\%)$ & $\begin{array}{l}\text { Trend Test } \\
p \text { value }\end{array}$ & Cause of Death & N (\%) & $\begin{array}{l}\text { Trend Test } \\
p \text { value }\end{array}$ \\
\hline $2014-2016$ & $31(7.52)$ & & $2014-2016$ & $43(14.14)$ & \\
\hline Mental disorders due to substance abuse & & 0.3142 & Mental disorders due to substance abuse & & 0.0061 \\
\hline $2002-2004$ & $57(8.64)$ & & $2002-2004$ & $22(6.61)$ & \\
\hline $2005-2007$ & $66(10.36)$ & & 2005-2007 & $33(9.51)$ & \\
\hline $2008-2010$ & $43(9.11)$ & & 2008-2010 & $21(7.58)$ & \\
\hline $2011-2013$ & $42(9.33)$ & & $2011-2013$ & $33(12.41)$ & \\
\hline 2014-2016 & $28(6.80)$ & & 2014-2016 & $38(12.50)$ & \\
\hline Non-AIDS cancer & & $<0.0001$ & Non-AIDS cancer & & 0.0003 \\
\hline $2002-2004$ & $79(11.97)$ & & $2002-2004$ & $31(9.31)$ & \\
\hline $2005-2007$ & $102(16.01)$ & & 2005-2007 & $47(13.54)$ & \\
\hline 2008-2010 & $77(16.31)$ & & 2008-2010 & $43(15.52)$ & \\
\hline $2011-2013$ & $88(19.56)$ & & 2011-2013 & $49(18.42)$ & \\
\hline 2014-2016 & $83(20.15)$ & & 2014-2016 & $56(18.42)$ & \\
\hline Overdose & & $<0.0001$ & Overdose & & 0.3463 \\
\hline 2002-2004 & $18(2.73)$ & & $2002-2004$ & $18(5.41)$ & \\
\hline $2005-2007$ & $47(7.38)$ & & 2005-2007 & $19(5.48)$ & \\
\hline 2008-2010 & $56(11.86)$ & & 2008-2010 & $21(7.58)$ & \\
\hline $2011-2013$ & $51(11.33)$ & & 2011-2013 & $23(8.65)$ & \\
\hline 2014-2016 & $50(12.14)$ & & 2014-2016 & $18(5.92)$ & \\
\hline Pancreatitis/ cancer & & 0.2176 & Pancreatitis/ cancer & & 0.5330 \\
\hline $2002-2004$ & $9(1.36)$ & & $2002-2004$ & $2(0.60)$ & \\
\hline $2005-2007$ & $2(0.31)$ & & 2005-2007 & $1(0.29)$ & \\
\hline 2008-2010 & $2(0.42)$ & & $2008-2010$ & $3(1.08)$ & \\
\hline $2011-2013$ & $2(0.44)$ & & $2011-2013$ & $0(0.00)$ & \\
\hline 2014-2016 & $3(0.73)$ & & 2014-2016 & $1(0.33)$ & \\
\hline Pneumonia & & 0.0045 & Pneumonia & & 0.0264 \\
\hline $2002-2004$ & $96(14.55)$ & & $2002-2004$ & $49(14.71)$ & \\
\hline $2005-2007$ & $81(12.72)$ & & 2005-2007 & $39(11.24)$ & \\
\hline 2008-2010 & $61(12.92)$ & & 2008-2010 & $39(14.08)$ & \\
\hline $2011-2013$ & $48(10.67)$ & & $2011-2013$ & $19(7.14)$ & \\
\hline 2014-2016 & 37 (8.98) & & 2014-2016 & $31(10.20)$ & \\
\hline Renal & & 0.4942 & Renal & & 0.4874 \\
\hline $2002-2004$ & $84(12.73)$ & & $2002-2004$ & $25(7.51)$ & \\
\hline $2005-2007$ & $65(10.20)$ & & $2005-2007$ & $40(11.53)$ & \\
\hline 2008-2010 & $48(10.17)$ & & 2008-2010 & $29(10.47)$ & \\
\hline 2011-2013 & $46(10.22)$ & & 2011-2013 & $22(8.27)$ & \\
\hline 2014-2016 & $48(11.65)$ & & 2014-2016 & $33(10.86)$ & \\
\hline Septicemia & & 0.3818 & Septicemia & & 0.6743 \\
\hline 2002-2004 & 81 (12.27) & & $2002-2004$ & $32(9.61)$ & \\
\hline 2005-2007 & 66 (10.36) & & 2005-2007 & $34(9.80)$ & \\
\hline 2008-2010 & $45(9.53)$ & & 2008-2010 & $28(10.11)$ & \\
\hline 2011-2013 & $47(10.44)$ & & 2011-2013 & $22(8.27)$ & \\
\hline
\end{tabular}


Table 2 Temporal trends in cause-specific deaths by county of residency at time of death (San Francisco County vs. all other counties), among people diagnosed with HIV in San Francisco who died in 2002-2016 (Continued)

\begin{tabular}{|c|c|c|c|c|c|}
\hline \multicolumn{3}{|c|}{ Cause of Death among San Francisco Residents } & \multicolumn{3}{|c|}{ Cause of Death among Non-San Francisco Residents } \\
\hline Cause of Death & $\mathrm{N}(\%)$ & $\begin{array}{l}\text { Trend Test } \\
p \text { value }\end{array}$ & Cause of Death & N (\%) & $\begin{array}{l}\text { Trend Test } \\
p \text { value }\end{array}$ \\
\hline $2014-2016$ & $44(10.68)$ & & 2014-2016 & $28(9.21)$ & \\
\hline Suicide & & 0.8411 & Suicide & & 0.2143 \\
\hline $2002-2004$ & $16(2.42)$ & & $2002-2004$ & $9(2.70)$ & \\
\hline $2005-2007$ & $24(3.77)$ & & $2005-2007$ & $12(3.46)$ & \\
\hline 2008-2010 & $22(4.66)$ & & 2008-2010 & $10(3.61)$ & \\
\hline $2011-2013$ & $14(3.11)$ & & $2011-2013$ & $12(4.51)$ & \\
\hline $2014-2016$ & $11(2.67)$ & & 2014-2016 & $13(4.28)$ & \\
\hline Viral hepatitis & & 0.0603 & Viral hepatitis & & 0.5918 \\
\hline $2002-2004$ & $108(16.36)$ & & $2002-2004$ & $50(15.02)$ & \\
\hline $2005-2007$ & $97(15.23)$ & & $2005-2007$ & $39(10.37)$ & \\
\hline 2008-2010 & $58(12.29)$ & & 2008-2010 & $32(11.55)$ & \\
\hline $2011-2013$ & 59 (12.89) & & 2011-2013 & $31(11.65)$ & \\
\hline 2014-2016 & 55 (13.35) & & 2014-2016 & 39 (12.83) & \\
\hline
\end{tabular}

$P$ values $<0.05$ are shown in bold

\section{Discussion}

We observed that PLWH who experienced homelessness were more likely to have mental disorders, and in particular mental disorders related to substance use, as a contributory cause of death, as hypothesized. We also saw a lower risk of death from non-AIDS cancer among homeless PLWH compared to those who were housed. There was no association with other causes of death between the housed and homeless population, including HIV/AIDS, non-cancer AIDS opportunistic infections, and AIDS cancer, suggesting that the benefit of access to early and sustained ART among PLWH was independent of housing status.

Both substance use and mental disorders are common among persons living with HIV and we observed deaths due to drug overdoses increased over time in San Francisco. Studies reported that approximately $40 \%$ of PLWH have some type of mental disorder, and $21-37 \%$ of PLWH experience substance use disorders [20, 21]. Substance use and mental disorders have been associated with decreased ART adherence and worse health outcomes [22-24]. PLWH with a history of injection drug use were reported to be less likely to access ART in several studies [25, 26]. Another study in Philadelphia observed that PLWH who had mental illness were less likely to achieve viral suppression than PLWH without mental illness (adjusted OR 0.65, 95\% CI 0.47, 0.91) [27]. These findings indicate that more outreach and/or services are needed to help those with mental health and substance use disorders.

Understanding how homelessness affects the health of PLWH who have co-occurring behavioral conditions is critical [28]. A study in New York City assessed whether the provision of supportive housing improves AIDS-free survival among chronically homeless PLWH with substance use or mental health disorders. The investigators observed a greater risk of death or AIDS diagnosis in unhoused compared to housed PLWH (adjusted hazard ratio $1.84,95 \%$ CI 1.40, 2.44) [29]. Because housing is a key social determinant of health for PLWH $[7,30,31]$ a continued investment in supportive housing is necessary to meet the U.S. End the HIV Epidemic goals [32].

Even in the absence of HIV, homeless individuals are more likely to need psychiatric services. Compared to housed patients, homeless patients with access to San Diego County mental health services were four times more likely to use hospitalization and emergency services to treat serious psychiatric issues [33]. This study also found that patients with schizophrenia and bipolar disorder were 1.6 and 2.4 times, respectively, more likely to be homeless when comparing to those with major depression [33].

The combination of homelessness, HIV, and mental illness is an area of synergy that requires more attention by health care providers and policy makers. There have been efforts to stabilize the marginally housed and improve mental health services in San Francisco through DAH, Care Not Cash, and MHSA. Additionally, in 2018, California voters passed Prop 2, which allows revenue generated from MHSA to be used specifically for housing homeless people in need of mental health services and San Francisco voters passed Prop C, which will tax businesses to generate funds for housing and homeless services. However, additional public programs may be 
Table 3 Unadjusted and adjusted Poisson regression prevalence ratios and 95\% confidence intervals for experiencing homelessness among 4158 people diagnosed with HIV in San Francisco who died in 2002-2016

\begin{tabular}{|c|c|c|}
\hline Outcome & Unadjusted PR (95\% Cl) for homelessness & Adjusted PR (95\% Cl) for homelessness \\
\hline Accident (including overdose) $(n=417)$ & $1.53(1.20,1.95)$ & $1.11(0.83,1.49)$ \\
\hline AIDS cancer ${ }^{a}(n=328)$ & $0.70(0.48,1.00)$ & $0.89(0.58,1.35)$ \\
\hline AIDS opportunistic infections (excluding AIDS cancers) ${ }^{a}(n=915)$ & $1.05(0.87,1.26)$ & \\
\hline Alcoholic liver disease $(n=47)$ & $1.32(0.62,2.83)$ & \\
\hline Assault $(n=32)$ & $2.52(1.17,5.44)$ & $2.56(0.90,7.28)$ \\
\hline Cardiomyopathy $(n=91)$ & $0.89(0.47,1.66)$ & \\
\hline Cerebrovascular disease $(n=141)$ & $0.71(0.41,1.23)$ & \\
\hline $\operatorname{COPD}(n=262)$ & $1.03(0.72,1.46)$ & \\
\hline Diabetes $(n=165)$ & $0.46(0.25,0.85)$ & $0.60(0.30,1.17)$ \\
\hline Heart disease ${ }^{a}(n=1033)$ & $0.86(0.71,1.04)$ & \\
\hline $\mathrm{HIV} \operatorname{AIDS}^{\mathrm{a}}(n=2928)$ & $0.95(0.85,1.06)$ & \\
\hline Ischemic heart disease $(n=311)$ & $0.59(0.39,0.88)$ & $0.87(0.55,1.38)$ \\
\hline Liver disease $(n=569)$ & $1.21(0.97,1.52)$ & \\
\hline Mental disorders ${ }^{\mathrm{a}}(n=427)$ & $2.15(1.73,2.78)$ & $1.63(1.24,2.14)$ \\
\hline Mental disorders due to substance use $(n=383)$ & $2.24(1.79,2.82)$ & $1.70(1.27,2.27)$ \\
\hline Non-AIDS cancer ${ }^{\mathrm{a}}(n=655)$ & $0.43(0.31,0.59)$ & $0.63(0.44,0.89)$ \\
\hline Overdose $(n=321)$ & $1.57(1.19,2.07)$ & $1.06(0.76,1.47)$ \\
\hline Pancreatic disease $(n=25)$ & $1.23(0.42,3.57)$ & \\
\hline Pneumonia $(n=500)$ & $1.12(0.87,1.43)$ & \\
\hline Renal disease $(n=440)$ & $1.12(0.86,1.45)$ & \\
\hline Septicemia $(n=427)$ & $1.14(0.87,1.48)$ & \\
\hline Suicide $(n=143)$ & $0.70(0.40,1.21)$ & \\
\hline Viral Hepatitis $(n=564)$ & $1.32(1.06,1.65)$ & $0.88(0.68,1.38)$ \\
\hline
\end{tabular}

Poisson regression adjusted for gender, race, age, concurrent HIV and AIDS diagnosis, HIV transmission risk, income status at diagnosis, year of death, prescription of ART, and San Francisco resident at time of death

${ }^{a}$ Composite causes of death

$P$ values $<0.05$ are shown in bold

needed to improve mental health services and substance abuse resources for homeless PLWH in particular.

Many studies have reported an increased incidence of non-AIDS cancer among PLWH due to increasing life expectancy and higher rates of co-infection with oncogenic viruses like Epstein Barr Virus, Human Papillomavirus, and Hepatitis B [34-39]. However, PLWH who were homeless in our study were less likely to die from non-AIDS cancers (such as lung, liver, anal, colon, pancreatic, rectal, leukemia, and Hodgkin lymphoma) most likely because of other competing causes of death, such as mental disorders and substance use. Another potential explanation is that people who are homeless are less likely to get diagnosed with cancer due to limited access to health care in general and cancer screening in particular.

There are several limitations to this study. First, the cause of death data, as noted on the death certificates, may be inaccurate or incomplete, an issue which has been shown to commonly occur in the United States
[40-42]. Inaccurate reporting would lead to misclassification bias and incomplete reporting would lead to imprecision. Second, information on housing status prior to HIV diagnosis was not recorded in the San Francisco HIV/AIDS registry; therefore, individuals who were homeless prior to being diagnosed with HIV were categorized as housed. Nevertheless, being homeless prior to HIV diagnosis likely had less of an influence on causes of death compared to any recent history of homelessness since diagnosis. Additionally, individuals who were homeless at the time of diagnosis and were subsequently housed were categorized as homeless, which would bias our results towards the null. Third, we did not have individual-level information on use of HIV and housingrelated programs. Instead, we used calendar time and county of residence at death as a surrogate to measure access to San Francisco and California specific HIV and housing interventions. We also did not have individuallevel data on ART adherence or discontinuation, but rather assumed that all deaths occurred in the era of wide 
ART availability. Last, the definition of homeless used by HIV surveillance excludes those who are marginally housed and thus combining these individuals with those who have stable housing might have diminished the impact of housing status on the various causes of death.

Despite these limitations, our study has several strengths. First, the use of cause of death data from death certificates was standardized and used in other studies and thus our results can be more easily compared. Second, the systematic collection of housing status both at the time of diagnosis and through subsequent medical chart reviews provide information that is unique and not widely available elsewhere. Third, the San Francisco HIV/AIDS surveillance registry was $95-99 \%$ complete $^{1}$ and thus is less subject to reporting bias. Last, the study was relatively large, population-based, and spanned a 15-year time period, all in the era of effective ART making our observations generalizable, comprehensive, and timely.

\section{Conclusions}

In summary, our analyses identified the life-threatening conditions and diseases that homeless people living with HIV may experience and these results can be used to direct policy decisions aimed at reducing mortality among this population. Such policies need to involve multiple approaches such as increased mental health services and housing in the homeless PLWH population. However, more research is needed to quantitatively evaluate use of specific mental health services provided in San Francisco, especially in light of the upcoming housing support for persons experiencing homelessness from the recently enacted State and local ballot propositions. Treatments for HIV have achieved remarkable success in preventing HIV-related deaths; as such the social determinants of health that still impact mortality must continue to be monitored and evaluated so that appropriate and effective policies and interventions can be implemented to reduce mortality among all persons living with HIV.

\section{Abbreviations}

AIDS: Acquired immunodeficiency syndrome; aPR: Adjusted prevalence ratio; ART: Antiretroviral therapy; CD4: CD4 T lymphocyte; CDC: Centers for Disease Control and Prevention; Cl: Confidence interval; DAH: Direct access to housing; DPH: Department of Public Health; HIV: Human immunodeficiency virus; HSC: Health and safety code; IRB: Institutional Review Board; MHSA: Mental Health Services Act; MSM: Men who have sex with men; MSMPWID: Men who have sex with men and who also inject drug; OR: Odds ratio; PLWH: People living with HIV; PR: Prevalence ratio; PWID: Person who injects drugs; RAPID: Rapid ART program initiative for new diagnosis; SFGH: San Francisco General Hospital

\section{Acknowledgements}

The authors would like to acknowledge the San Francisco Department of Public Health surveillance staff for data collection.

\section{Authors' contributions}

$\mathrm{NH}$ was a major contributor in writing the manuscript and performing statistical analyses of the data. ME was a major contributor in writing the manuscript. SP, $\mathrm{LH}$, SS had substantively revised the manuscript. AV generated the dataset from the HIV surveillance registry and substantively revised the manuscript. All authors have read and approved the final manuscript.

\section{Funding}

This study was partially supported by the Centers for Disease Control and Prevention PS13-1302, Grant number SU62PS004022-04, Core and Incidence HIV Surveillance. The funder played no role in the design of the study, or the collection, analysis, and interpretation of data, or in the writing of the manuscript.

\section{Availability of data and materials}

The datasets generated and/or analyzed during the current study are not publicly available as these HIV surveillance data are protected under California state law HSC 121022 and HSC 120130.

\section{Ethics approval and consent to participate}

HIV surveillance data collection is required by state law (California HSC 121022 and HSC 120130), therefore IRB approval and consent was deemed unnecessary according to state regulations. Data used in this study is protected by state law and is not publicly available.

\section{Consent for publication}

Not Applicable

\section{Competing interests}

The authors declare that they have no competing interest.

\section{Author details}

${ }^{1}$ Department of Clinical Pharmacy, University of California, 3333 California Street, Suite 420, San Francisco, CA 94118, USA. ²Department of Public Health, 25 Van Ness Avenue, Suite 500, San Francisco, CA 94102, USA.

Received: 16 July 2019 Accepted: 21 October 2019

Published online: 01 November 2019

\section{References}

1. San Francisco Department of Public Health. HIV epidemiology annual report 2017; Available at https://www.sfdph.org/dph/comupg/oprograms/ HIVepiSec/HIVepiSecReports.asp. Assessed 10 Nov 2018.

2. Housing Instability Research Department. San Francisco homeless count and survey comprehensive report. http://hsh.sfgov.org/wp-content/ uploads/2017/06/2017-SF-Point-in-Time-Count-General-FINAL-6.21.17.pdf. Accessed 6 June 2018.

3. Hibbs JR, Benner L, Klugman L, et al. Mortality in a cohort of homeless adults in Philadelphia. N Engl J Med. 1994;331(5):304-9.

4. Barrow SM, Herman DB, Cordova P, Struening EL. Mortality among homeless shelter residents in New York City. Am J Public Health. 1999:89(4): 529-34.

5. Baggett TP, Hwang SW, O'Connell JJ, et al. Mortality among homeless adults in Boston: shifts in causes of death over a 15-year period. JAMA Intern Med. 2013;173(3):189-95.

6. Khanijow K, Hirozawa A, Ancock B, Hsu LC, Bamberger J, Schwarcz SK. Difference in survival between housed and homeless individuals with HIV, San Francisco, 2002-2011. J Health Care Poor Underserved. 2015;26(3):1005-18.

7. Schwarcz SK, Hsu LC, Vittinghoff E, Vu A, Bamberger JD, Katz MH. Impact of housing on the survival of persons with AIDS. BMC Public Health. 2009;9:220.

8. Clemenzi-Allen A, Geng E, Christopoulos K, et al. Degree of Housing Instability Shows Independent "Dose-Response" With Virologic Suppression Rates Among People Living With Human Immunodeficiency Virus. Open Forum Infect Dis. 2018;5(3):ofy035.

9. Direct access to housing (DAH). Department of Homelessness and Supportive Housing Web site. http://hsh.sfgov.org/wp-content/uploads/201 7/08/DAH-Information.pdf. Accessed 6 June 2018.

10. Tipton M, Alderete P, Flaherty S. Human services agency: Care not cash is achieving its goals. City and County of San Francisco Web site. https://www. sfcontroller.org/ftp/uploadedfiles/controller/reports/CareNotCash_20080430. pdf. Updated 2008. Accessed 5 Jan 2018.

11. Proposition 63. Mental health services expansion and funding. Legislative Analyst's Office Web site. https://lao.ca.gov/ballot/2004/63_11_2004.htm. Updated 2004. Accessed 6 Jan 2018. 
12. Douglas T. Mental health services act (MHSA) housing program semi-annual update. Department of Health Care Services Web site. https://www.dhcs.ca. gov/services/MH/Documents/4-2013SemiAnnRpt.pdf. Updated 2013. Accessed 6 Jan 2018

13. Zetola NM, Klausner JD, Haller B, Nassos P, Katz MH. Association between rates of HIV testing and elimination of written consents in San Francisco. JAMA. 2007;297(10):1061-2.

14. Schwarcz S, Hsu LC, Scheer S. Disparities and trends in viral suppression during a transition to a "test and treat" approach to the HIV epidemic, San Francisco, 2008-2012. J Acquir Immune Defic Syndr. 2015;70(5):529-37.

15. San Francisco program for rapid ART initiation and linkage to care: Standard operating procedures. San Francisco Department of Public Health Web site. https://www.gettingtozerosf.org/wp-content/uploads/2016/09/20160822_ citywide_rapid_protocol_v2.pdf. Accessed 9 Jan 2018.

16. Centers for Disease Control and Prevention. International Classification of Diseases, Tenth Revision, Clinical Modification (ICD-10-CM). Hyattsville: National Center for Health Statistics; 1999.

17. Centers for Disease Control and Prevention. Revised surveillance case definition for HIV infection-United States, 2014. MMWR Recomm Rep. 2014; 63(RR-03):1-10.

18. U.S. Census Bureau, 2006-2010 American community survey: selected economic characteristics. Washington, D.C.: U.S. Department of Commerce.

19. SAS Institute Inc, editor. ed SAS/STAT User's Guide, Version 9.4: Cary, SAS Institute Inc; 2014.

20. Bing EG, Burnam MA, Longshore $D$, et al. Psychiatric disorders and drug use among human immunodeficiency virus-infected adults in the United States. Arch Gen Psychiatry. 2001;58(8):721-8.

21. Pence BW, Miller WC, Whetten K, Eron JJ, Gaynes BN. Prevalence of DSM-IVdefined mood, anxiety, and substance use disorders in an HIV clinic in the southeastern United States. J Acquir Immune Defic Syndr. 2006;42(3):298-306.

22. Chander G, Himelhoch S, Moore RD. Substance abuse and psychiatric disorders in HIV-positive patients: epidemiology and impact on antiretroviral therapy. Drugs. 2006;66(6):769-89.

23. Ingersoll K. The impact of psychiatric symptoms, drug use, and medication regimen on non-adherence to HIV treatment. AIDS Care. 2004;16(2):199-211.

24. Herbeck DM, Fitek DJ, Svikis DS, Montoya ID, Marcus SC, West JC. Treatment compliance in patients with comorbid psychiatric and substance use disorders. Am J Addict. 2005;14(3):195-207.

25. Strathdee SA, Palepu A, Cornelisse PG, et al. Barriers to use of free antiretroviral therapy in injection drug users. JAMA. 1998;280(6):547-9.

26. Gebo KA, Fleishman JA, Conviser R, et al. Racial and gender disparities in receipt of highly active antiretroviral therapy persist in a multistate sample of HIV patients in 2001. J Acquir Immune Defic Syndr. 2005;38(1):96-103.

27. Yehia BR, Stephens-Shield AJ, Momplaisir F, et al. Health outcomes of HIVinfected people with mental illness. AIDS Behav. 2015;19(8):1491-500.

28. Leaver CA, Bargh G, Dunn JR, Hwang SW. The effects of housing status on health-related outcomes in people living with HIV: a systematic review of the literature. AIDS Behav. 2007;11(6 Suppl):85-100.

29. Hall G, Singh T, Lim SW. Supportive housing promotes AIDS-free survival for chronically homeless HIV positive persons with behavioral health conditions. AIDS Behav. 2019;23(3):776-83.

30. Buchanan D, Kee R, Sadowski LS, Garcia D. The health impact of supportive housing for HIV-positive homeless patients: a randomized controlled trial. Am J Public Health. 2009;99(Suppl 3):S675-80.

31. Milloy MJ, Marshall BD, Montaner J, Wood E. Housing status and the health of people living with HIV/AIDS. Curr HIV/AIDS Rep. 2012;9(4):364-74

32. Fauci AS, Redfield RR, Sigounas G, Weahkee MD, Giroir BP. Ending the HIV epidemic: a plan for the United States. JAMA. 2019;321(9):844-45.

33. Folsom DP, Hawthorne W, Lindamer $L$, et al. Prevalence and risk factors for homelessness and utilization of mental health services among 10,340 patients with serious mental illness in a large public mental health system. Am J Psychiatry. 2005;162(2):370-6.

34. Patel P, Hanson DL, Sullivan PS, et al. Incidence of types of cancer among HIV-infected persons compared with the general population in the United States, 1992-2003. Ann Intern Med. 2008;148(10):728-36.

35. Silverberg MJ, Chao C, Leyden WA, et al. HIV infection and the risk of cancers with and without a known infectious cause. AIDS. 2009;23(17):2337-45.

36. Crum-Cianflone NF, Hullsiek KH, Marconi V, et al. Trends in the incidence of cancers among HIV-infected persons and the impact of antiretroviral therapy: authors' reply. AIDS. 2009;23(13):1791-2.
37. Shiels MS, Cole SR, Kirk GD, Poole C. A meta-analysis of the incidence of non-AIDS cancers in HIV-infected individuals. J Acquir Immune Defic Syndr. 2009;52(5):611-22.

38. Grulich $A E$, van Leeuwen MT, Falster MO, Vajdic CM. Incidence of cancers in people with HIV/AIDS compared with immunosuppressed transplant recipients: a meta-analysis. Lancet. 2007;370(9581):59-67.

39. Lima VD, Hogg RS, Harrigan PR, et al. Continued improvement in survival among HIV-infected individuals with newer forms of highly active antiretroviral therapy. AIDS. 2007;21(6):685-92.

40. Smith Sehdev AE, Hutchins GM. Problems with proper completion and accuracy of the cause-of-death statement. Arch Intern Med. 2001;161(2): 277-84

41. Trepka MJ, Sheehan DM, Fennie KP, Niyonsenga T, Lieb S, Maddox LM. Completeness of HIV reporting on death certificates for Floridians reported with HIV infection, 2000-2011. AIDS Care. 2016;28(1):98-103.

42. McGivern L, Shulman L, Carney JK, Shapiro S, Bundock E. Death certification errors and the effect on mortality statistics. Public Health Rep. 2017;132(6): 669-75.

\section{Publisher's Note}

Springer Nature remains neutral with regard to jurisdictional claims in published maps and institutional affiliations.
Ready to submit your research? Choose BMC and benefit from:

- fast, convenient online submission

- thorough peer review by experienced researchers in your field

- rapid publication on acceptance

- support for research data, including large and complex data types

- gold Open Access which fosters wider collaboration and increased citations

- maximum visibility for your research: over $100 \mathrm{M}$ website views per year

At $\mathrm{BMC}$, research is always in progress.

Learn more biomedcentral.com/submissions 